\title{
Algorithmic Governance in Smart Cities: the Conundrum and the Potential Pervasive Computing Solutions
}

\author{
Franco Zambonelli, Università di Modena e Reggio Emilia, Italy \\ <franco.zambonelli@unimore.it>, \\ Flora Salim, RMIT University, Australia \\ <flora.salim@rmit.edu.au >, \\ Seng W. Loke, Deakin University, Australia \\ <seng.loke@deakin.edu.au>, \\ Wolfgang De Meuter, Vrije Universiteit Brussel, Belgium \\ <wdmeuter@vub.ac.be>, \\ Salil Kanhere, University of New South Wales, Australia \\ <salil.kanhere@unsw.edu.au>
}

\begin{abstract}
Smart cities and smart environments - as enabled by pervasive computing technologies - can make us become smarter, relieve us from many customary activities and even take over some boring and repetitive cognitive activities. However, delegating too many decisions to the environment may eventually make us lose attention and make us become "dumber", or worse, make us lose control over our activities and lead to all kinds of "algorithmic governance". In this article we discuss the problems this can potentially bring at individual and societal levels, and eventually analyze some key directions to attack these potential problems and - via the help of the same pervasive computing technologies - turn them into advantages.
\end{abstract}

Index Terms - Algorithmic governance, grey-box, context-aware systems, smart cities

\section{INTRODUCTION}

Pervasive and mobile computing technologies can make our everyday living environments and our cities "smart", i.e., capable of reaching awareness of physical and social processes and of dynamically affecting them in a purposeful way [1]. This is already happening, e.g., in the form of digital traffic signs that suggest in real-time the best traffic directions or the availability of parking spots, and also in the form of locationbased social networks that inform us about noteworthy events. Soon, we expect that the pervasive diffusion of sensing, actuation, and computing, will allow our urban environment to fully self-regulate in autonomy most of its processes, and to guide and support our everyday activities.

It is generally acknowledged that living in a smart environment makes us smarter, by increasing our overall levels of awareness of the ongoing urban activities [2]. Also, by supporting and facilitating our customary activities (e.g., driving, finding information and goods), living in a smart environment can make life much more pleasant and less stressful, and also make the environment more sustainable.

However, the evolution of smart environments also carries potential risks for individuals and for society as a whole. In particular, if most of our everyday activities can be automated, we could be tempted to increasingly delegate the governance of such activities, and the governance of the whole city, to the 
algorithmic engines of the smart city infrastructure. Thus, rather than taking advantage of the augmented capabilities of perception and participation enabled by the technologies, we could end up losing critical attention, abandoning individual decision making for relying on collective computational governance of our activity, losing awareness of environmental and social processes, and ultimately lose power and become dumb [3].

In this article, we elaborate on the key concepts of algorithmic governance in smart cities, discussing its likely increasing role in the future. Without any intention of dramatizing or of embracing dystopian visions, we intend to outline some specific problems that could potentially occur in future smart cities, and eventually analyse some key directions to prevent or mitigate - also with the fundamental support of pervasive computing technologies - these potential problems and possibly turn them into advantages.

\section{SMART Cities: From Citizen Support to Algorithmic Governance}

Algorithmic governance, in general terms, concerns empowering software to take decisions and to autonomously - i.e., without human supervision - regulate some aspects of our everyday human activities or some aspect of the society, according to some algorithmically defined policies [4]-[6].

We are already subject to algorithmic governance in a variety of different aspects of our personal and social life. Google search dictates what information we find on the Internet, as we typically accept the suggestions appearing on the first page. Facebook news feed algorithms dictates what are the relevant posts to show us, and given that young people rely on Facebook as the primary source of news, this implies that they have fully delegated to Facebook the activity of seeking and filtering information [7].

Moving from the individual to the societal sphere, examples of algorithmic governance can be found in trading, where most of decisions (and thus the oscillations of markets and our finances) now rely on complex agent-based decision making; or in the pricing strategies of airlines that rely on complex analysis of travel trends.

In the area or urban management, some early examples of algorithmic governance can be found in traffic management (e.g., traffic lights that adaptively their frequency depending on the sensed traffic flow), public transport (e.g., to adapt bus schedule and routes to meet the transport demand in real time) and energy management (e.g., to automatically tune energy pricing depending on the instantaneous balance between supply and demand).

Current examples of algorithmic governance for smart cities still rely on rather limited capabilities of sensing and actuating. However, the increasing spread of pervasive computing and IoT (Internet of things) technologies will soon make it possible to sense at incredible levels of detail every single event happening in every corner of a city, and to trigger flexible actions to affect the state of things via a variety of actuators, robots and autonomous vehicles [1]. The assessed mid-term future for urban mobility is the one in which citizens and merchandise will be carried to any desired destination via myriads of self-driving vehicles, globally orchestrating their movements and routes with each other and with the urban street infrastructure. Similarly, the flow of pedestrians will be somehow steered (via digital signages or apps on wearable devices) and orchestrated so as to avoid dangerous situations [8].

Pushing the vision forward, we can imagine that the entire management of cities will be soon governed and actuated in an automatic, unsupervised way. For instance, this can include the management of waste collection (which also includes the possibility to individually account citizens for what they produce as waste), decisions on new urbanization, management of roads and other infrastructures (e.g., by automatically deciding which roads and traffic lights to replace depending on their actual state and available budget).

In general, living in a smart environment and being made part of its awareness can potentially make us smarter. That is, it can notably increase our perceptory and social capabilities [2], and our capabilities of understanding situations and reacting to them. In addition, letting our everyday urban life be governed by 
some automated software systems, promises to notably increase our quality of life. In fact, it will relieve us from a number of boring physical and mental activities, and enable us to do much more interesting things that, say, driving and having to decide how to head to the destination. In other words, it will make it possible to satisfy needs at the highest levels of the Maslow pyramid [9]. However, as we will elaborate in the following, algorithmic governance also comes with a number of potential dangers, and is at risk of somehow making us "dumber" rather than smarter.

\section{The Possible Perils of Algorithmic GovernANCE}

The primary source of all perils related to algorithmic governance is that, very often, software and algorithms are designed as "black boxes" with little understanding of how they actually work. Such little understanding not only involves the final users (i.e., in case of smart cities, all citizens) but quite often also the stakeholders (e.g., municipalities and decision makers) and the developers, if they do not care enough to understand how these algorithms operate and have a discussion with designers and stakeholders.

This limited understanding of algorithms, for what we can see so far, does not prevent people from relying on them for several activities. For instance, people fully rely onis Google's results even without knowing anything about its underlying PageRank algorithms. This is the key difference between algorithms and other classes of technologies: using them and relying on their judgement and suggestions without knowing the reasons we are being suggested something. In smart cities, a pervasive environment governed by algorithms will make us apparently smarter in our capabilities, but to some extent will also make us somehow "dumber" in that our actions will no longer be conscious. For instance, roaming in an unknown city with a paper map enable us to catch the basic fabric of the city and, on this basis, to consciously decide what route to take. Conversely, roaming with a GPS navigator does not require knowing anything about the city structure, and we are willing to accept route suggestions without investigating further on the reason a certain route has been selected. In other words, our notion of "dumber" here in in the sense of potentially losing the ability to make good judgement in some situations, reducing discernment, and lazily deferring to the algorithm. The over-reliance on algorithms, which are capable (unlike other technologies) to make judgements or decisions on our behalf without questioning and thinking through them, will make us increasingly depend on them for our everyday life, instead of depending on human rationale and our own reasoning.

One may say that the above issue would not cause any trouble, because algorithms will be developed for the sake of serving citizens, for their own good and in accord to the rules and policy of the municipality, and that such rules will be made transparent. Yet, as we elaborate in the following: (i) decisions made by algorithms may be biased and have inaccurate information; (ii) there is risk that someone manage to make intentional misuse of algorithms without no one noticing; (iii) politics and decision-makers themselves may end up having little clue on the actual working of algorithms, thus losing power.

With regard to the first issue, we normally trust the developers of the technologies we use, knowing that societal norms, regulations, and reputation largely help to protect that trust. However, intentional or (more often) unintentional bias, errors, incomplete information, can subtly hide in algorithms, along with bias in behavior dues to values (right or wrong) that developers or creators of the technology might have. This issue could be exacerbated by the increasing influence and role of technology in daily decision-making. For example, bias can creep into a machine learning algorithm [10] due to wrong or inadequate datasets, not any malice of the developers (e.g., the system that is biased against people with black names when judging potential for crime, or software which could not do face recognition well for people with dark sin, or unfair judgments on insurance or loans, all due to issues with data used in training the algorithms). Also, what is a "normal person" or "normal behavior" is often contextual and hard to define - which can influence default settings in software [11], e.g., gender bias in default character profiles in some games, or someone struggling to read a critical alert message in a non-native language, or what an algorithm portrays as 
"normal looking".

Algorithms also could have incomplete information in decision-making or lack knowledge of exceptional cases even if developers do endeavor to be comprehensive. In some cases, even if tempted to doubt the algorithm, the profitability to follow the algorithm's decision can be high, not necessarily by virtue of how good the algorithm is. For instance, with smart algorithms performing sentencing, or the "robotization of justice" [12], by deciding against an algorithm's position for guiltiness one take personally the responsibility of a possible second offence. In any case, even if the thought that algorithms can do better than humans is becoming prevalent, and despite the recent breakthroughs in deep learning, for neural networks to achieve the computational level of a human brain it still needs far more power [13].

With regard to the second issue, independently of the reliability and trustability of algorithms, there is the issue of who is devoted to manage such algorithms and of how we can trust such people in managing the algorithms. For instance, developers could also be influenced by imperatives from (e.g., an abusive) government, when developing technology. If there is a problem with current government, algorithmic technology could be intentionally misused, hiding behind the "veil of sophistication". In particular, if algorithms are left to their own devices to govern smart cities, the more complex conundrum is how algorithmic government models for smart cities should look like to prevent or mitigate voluntary or unintended abuses. Who will be charge of deciding which algorithms to run? If politics are in charge of deciding the rules governing a city, who will be in charge of developing algorithms and check they are adherent to the rules? Who will tune the parameters for the algorithms and how? What about the possibility of instantiating multiple algorithms, and that such algorithms become agonistic [14], i.e., conflicting with each other? What does an objective function for the governance models look like? What does the termination condition for algorithmic governance models look like? Who will be responsible for bugs? For algorithms governing safety critical situations ${ }^{1}$, such questions have already became pressing, and this problem will dramatically exacerbate in the future. Just think at the use of AI and autonomous decisions in weaponry [15]. Hinton, in [13], also argued there there is not only need to tame AI research, but also to improve political systems so that AI is not going to be misused.

Strictly related, algorithms governing our cities may also be involved in decisions involving ethical or moral dilemmas. For instance, a human driver that managed to swerve her car in time to avoid killing several pedestrians while sacrificing herself might be lauded, but a self-driving car that killed its driver even while saving pedestrians might worry passengers [16]. Self-driving cars could reduce the need to learn driving - less people might know how to drive - which can be a problem for situations where such a skill is indeed needed, but moral algorithms that make human acceptable judgements are problematic. An algorithm that behaves in a utilitarian manner could benefit society as a whole but hurt individuals and might not be accepted.

With regard to the third issue, the risk that politicians and governors lose control over algorithms, can lead to an "algocracy". The term "algocracy", in contrast with "democracy", literally means that the power ("kratos" in greece) lies with the algorithms rather than with the people ("demos" in Greece). To some extent, it may appear we are already living in a partial algocracy, given that algorithmic decisions already affect some of our civil life. For instance, when applying for a visa for some countries, based on some business rules, applicants with a certain type of passport will receive immediate clearance, whereas others will be pushed to the next level of scrutinization, treating people differently based on settings and semantics of algorithms. Yet, until the settings and the semantic of such algorithms are perfectly compliant to legislation and rules, the power still resides with the government and, ultimately, with the people who voted them. However, given that algorithms can be difficult to implement, configure, and fully understand, the risk exists that governors end up relying on algorithms they do not fully understand, without being capable

${ }^{1}$ https://spectrum.ieee.org/computing/software/automated-to-death 
of effectively verifying the adherence of the algorithms to the existing laws. Thus, we may end up implicitly delegating decisional power to the algorithms, or to the bunch of people devoted to design and develop them.

Algorithms in future society and cities will serve the same role that civil law and urban regulations, respectively, have in today's democracy. Accordingly, new political procedures are to be put in place to regulate which code is installed, and the responsibility for (technical) code verification and (juridical) semantic verification will have to be clearly defined. Most importantly, to avoid algorithmic governance to degenerate into an algocracy, it will be necessary for citizens, politics, and decision makers, to become capable of understanding and harnessing the complexity of algorithms and their configuration.

\section{HOW TO DEAL WITH THE PROBLEMS}

Let us know analyze what solutions, possibly enabled by the same pervasive computing technologies that cause them, can be envisioned to attack the identified problems.

\section{A. Data Access Control}

Algorithmic governance in smart cities is enabled by the availability of large amounts of data, making it possible for algorithms to understand what is happening and act accordingly. In this context, the dense and pervasive collection, processing and dissemination of data in the midst of people's private lives, while useful to offer a range of sophisticated and personalized services that provide utility to the users, necessarily gives rise to specific privacy and algorithmic concerns.

From the privacy viewpoint, the pervasive collection of information exacerbates the existing issues associated with privacy in data handling. In fact, such find-grained can be used to algorithmically construct a virtual biography of our activities, revealing private behavior and lifestyle patterns. Disclosure of some of these unintended inferences gives rise to the notion of behavioral privacy [17] which is distinctly different from traditional identity privacy. The possession of such detailed personal information about an individual may confer power over that individual, resulting in potential misuse by governments, corporations, or other individuals. For example, households are being equipped with smart meters to act as providers of temporally fine-grained energy consumption reports. The utility companies use the data to better estimate the domestic power consumption leading to optimized distribution. However, as shown in [18], several unintended and sensitive inferences such as occupancy and lifestyle patterns of the occupants can be made from the data. Accordingly, we need configurable privacy-preserving tools that afford users fine-grained control over how their personal information is shared.

From the algorithmic viewpoint, it is of fundamental importance for users to understand what type of personal information is used by algorithms to take decisions, and how such information is used. And, in case the user understands that such information is not correct, biased, or not appropriately exploited (and thus leading to incorrect algorithmic behaviors) exploit the above-mentioned privacy-preserving tools to tune the usage of information by algorithms. For instance, consider an automated home heating system that self-regulate based on the life patterns of inhabitants (and accounts bills accordingly), and a person that was constrained at home for 15-days due to a bad winter flu. When such person eventually goes back to work, he should be able to "see" if the heating system is still acting on the basis of the wrong assumption that he is at home with a flu. The risk, otherwise, is to lazily (and stupidly) accept to pay more for heating.

For both concerns, pervasive computing technologies can potentially enable users to access the sensors around (e.g., in a wireless way via its mobile phone) and see what data they produce and how they what algorithms.

\section{B. Algorithmic Guardians}

If today algorithms affect what we can view online, tomorrow they'll modify our physical reality at home or in a smart city, and - as stated earlier - will do that in personalized way. However, even if we are given 
the possibility to see what data is being used and by what algorithms, the resulting personalization process might not be transparent or comprehensible, and could be hard to understand and interpret especially for non-data-literate users.

Beside the problem of understanding personalization, another issue that may arise concerns the fact that personalization might not always serve the users' interest, but rather the interests of the algorithms' creator. There is usually an interest gap between you and the third party that paid for the algorithms to prioritize something for you, which can lead to conflicts and obtrusive personalization. Different digital environments serve different interests and thus capture different areas of preferences. Algorithms generalize and simplify, as they continuously filter out things that are considered to be irrelevant and useless. In many cases, algorithms use other people's data to fill in missing bits and pieces.

Today our algorithmic selves are beyond our control and can leave us vulnerable. A possible solution could to be have software tools and algorithms that would be on our side and in our control — algorithmic guardians - capable of somehow protecting us from undesirable behavior of third party algorithms. We envision algorithmic guardians as far more evolved instances of current personal assistants such Siri, Watson, etc., that, thanks to wearable and advanced human-computer interactions models enabled by pervasive computing, will be always and easily accessible to interact with. Our digital guardian will protect us from algorithmic manipulation that restricts personal freedom and will make sure that we are not stuck on repeating behavioral loops or virtual echo-chambers. It will create an adaptive information interface that is fresh and relevant. Furthermore, guardian will support us in controlling our personal data flows and deciding who can access our digital trails. For instance, with reference to the home heating example of the previous sub-section, our personal guardian should be able to alert us that the heating systems is still acting as if we would have a flu and help us correcting such behavior.

Our digital guardian does not need to be intelligent in the same way as we humans. It would need to be smart in relation to the environments it inhabits - to other algorithms it would encounter. In any case, even if algorithmic guardians (unlike third party algorithms) will be owned by ourselves and will be totally under our own control, being able to understand how they work will be a priority to make them fully trustful. This issue, which applies to all algorithms that will govern our life - is elaborated in the following.

\section{Democracy through Grey-box Code for Algocracy}

Algorithms are created by a handful of people or, in a probable near future, by other algorithms which are created by an even smaller number of people. This raises an enormous challenge for our democracies.

In the spirit of "freedom of information", publishing the algorithms towards citizens seems like an obvious thing to do. However, this doesn't make a lot of sense if the overwhelming majority of the citizens cannot even read them. In today's democracy, civil law is also produced by a handful of specialists and to use it, we rely on lawyers. Nevertheless, the civil code can be consulted by anyone who is willing to make the mental effort of digging through some heavy prose. With the current literacy level of the general public in computer science, there is no analogue for this whatsoever: when rules and regulations would be expressed in code, only a tiny fraction of today's society is even able to read them.

There is compulsory need, for both citizens and governors, be able to code the rules that govern the algocracy in a format that is understandable to policy makers and to the public, also to make sure that we will be able to understand whether the code is actually serving the purposes it has been built for, or it is instead bugged or hacked. With this regard, we envision three key requirements: better programming languages, inspectability of code, and users' computing literacy.

Concerning programming languages, we emphasize that future algorithmic governance for smart cities, will be inherently distributed and mobile. Accordingly, its programming will require much better languages that we currently have for distributed programming where, for instance, there is little support for the verification of the corrent behavior of programs. This is confirmed by the tremendous number of middlewares that exist to cover their shortcomings, yet without integrating well with the host language 
[19]. Powerful languages that allow complex distributed code to be written in a "clean" way (such as e.g. AmbientTalk [20]) have not yet made it to the mainstream. What makes a "good" language for this job? An important yardstick to measure the quality of a language can be found in Brooks' paper on the complexity in software engineering [21]. Today's programming languages put far too much emphasis on the accidental complexity of a distributed system. Languages that allow a distributed programmer to only focus on the essential complexity are still being researched as we speak.

For inspectability, algorithms are often referred to as "black boxes" is that it is not apparent to the casual observer exactly how the algorithm works. Tomorrow's intelligent environments and algocracies should be based on "grey box" systems that citizens can read and tweak along various "levels" of participation. Just like there is a distinction between a constitution and normal laws, distinctions have to be made between various levels of code such that some code is easily tweakeable by direct democratic processes (à la WikiPedia) whereas other code is proverbially carved in stone. In other words, the code that runs the algocracy needs to be exposed in a "grey box" fashion where different shades of grey will probably be needed. For instance, in the area of pervasive computing and the Internet of Things, approaches to userlevel programming for configuration of smart environments, based on simple and understable "if this then that" rules (see e.g., www.ifttt.com) and hiding more mundane programming details, go in that directions. However, in a broader perspective, societal engagement would also include building "institutions and tools that put the society in-the-loop of algorithmic systems, and allows us to program, debug, and monitor the algorithmic social contract between humans and governance algorithms" [22]. The need for transparency, accountability, and explainability for the increasingly prevalent AI "black-boxes" has been noted in [23], where a layered model involving technical, ethical, legal and social aspects need to be taken into account.

In parallel with the development of an understandable "gray box" approach to programming, we need to solve one of the main hampering factors for a healthy algocracy: the need for citizens to have at least a basic literacy in computing, and - if not able to program - capable of judging the actions and the quality of the programs that govern them (at least when exposed in their "gray box" form). Unfortunately, computer science as a basic scientific field is absent in the high school systems of most countries. A notable exception is the UK where "Computing" is a part of the high school curriculum since 2015. Even for people that will never program in their entire life, a good basic understanding of what is programming and what is an algorithm is necessary for being a citizen in the algocracy!

\section{Humans in the Loop and the Wisdom of Many}

An algorithm is weightless and only worth the weight people put on it, and some degree of safety from the potential dangers of algorithmic governance can come from the "wisdom of the crowd". There is a need for users to be able to provide feedback to the system in a forum, or through a mechanism for collectively comment on the algorithm performance, so that problems can be identified and signaled. And the same pervasive sensing technologies that feed the algorithms with data can be exploited by users to monitor the environment and signals (and share information about) problems caused by existing smart city algorithms. For instance, the fact that home heating systems are biased so that they do not meet user needs and only act toward some municipality goals, can be discovered by a multitude of users (or by their algorithmic guardians) by accessing sensors and actuators data, and eventually can make the fact emerge to global awareness. Indeed, it should be a general goal for governments (at all levels, from national to municipal), whenener they start relying on algorithms to control cities and make decisions on our behalf, to involve human citizens in the loop at the highest levels of the participation ladder [3], i.e., as a partner, delegation authority, and co-manager of the system or algorithm.

A different possible form of the wisdom of the crowd can be the wisdom of the crowd of algorithms, enabled by the existence of a plurality of algorithms and systems devoted to govern the same concern. In essence, human decision might be based on some aggregation of the inputs of a number of preferably independent algorithms, that is, we can take the principle of the "wisdom of the many" to systems. 
However, when algorithms deals with ethical choices, the involvement of humans may become necessary. Should there be no time to involve a human in the decision-making, one can consider that some algorithmic decisions are premade by a human in advance so that human accountability is retained, and automatically adapted to the specific context once these decisions have to become actions.

Lastly, when there are systematic fails, there needs to be a way to pull the plug. Humans should be enabled to "pull the plug" on the algorithm - when it is found to deliver unfavourable outcomes or cause problems, humans can stop using the system. As a simple example, a user (as it is already indeed in our homes) should always be enable to turn off the automatic home heating system and go manual. How to control these systems and pull the plug needs to be obvious [24]. However, for critical systems, the look and feel of the control switches need to be different, so that unintentional, potentially fatal, mistakes are not made. As told in [24], the control-room operators in a nuclear power plant found that the similar-looking knobs could lead to a disastrous outcome, hence beer-keg handles are placed over them. Putting humans in ultimate control of algorithms would seem sensible, but is not without its own issues - e.g., a human cannot simply switch off an autonomous vehicle when s/he thinks it is not performing up to its requirements there is a need to deliver control back to humans safely, once in control, for humans to safely control the algorithm.

\section{E. From Context-awareness to Context-control-awareness}

Pervasive computing technologies enable algorithms to take decisions that are context-aware, i.e., adapted to the context in which they operate (the already mentioned personalization being a specific form of context-awareness). Context-awareness has been studied since the $90 \mathrm{~s}$ in the area of pervasive computing [25] [26], and the last decade has seen a dramatic progress in automatic recognition of context (including place - outdoors and indoors, human activity, habit, preference, and the available energy and resources, etc.) and the self-adaptation of pervasive computing devices to the learned context.

Given the possible perils of algorithmic governance, one possible research direction is "context-controlaware" systems. That is, algorithms that can override control are not given full access for adapting the devices under their influence but are, instead, given a shared access control with networked of socially connected devices, with humans as co-decision makers, for shared governance [27]. This allows pervasive computing systems to be more "considerate", as they are not only aware of their contexts (and input to the systems), but also how their influence and control (and output of the systems) can bring unintended consequences. Such shared control should also account for safety, security, and privacy that - although extensively researched so far mostly in a separate silo - have instead to be integrated in context-controlaware systems for safer smart environments.

The issue of control (and, strictly related, the previously mentioned issue related to "pulling the plug" mentioned) involved that of making smart cities and environments really usable, i.e., alluding to Steve Krug's attributes of usability of an interface [28], useful, learnable, memorable, desirable, and delightful. In particular, the studied contexts in context-control-aware computing for smart cities and environments should also provided with interfaces allowing the citizens and the end users to voice out their discomfort and displeasure on the systems, enabling higher-levels of interactivity with the governing algorithms, and enabling these systems to, e.g., 'reverse actions', 'pull back' and activate 'dumb mode' when required. We need to allow smart cities not just be "efficient", but also to make it possible for citizens to be more interactional with the governs of cities, and viceversa [29].

There is a need for balance between a system being too obtrusive to be useful so that the user is involved too often, versus a system that is too autonomous so that algorithmic regulation becomes real; a question is whether an algorithm be designed to compute this balance, i.e., to so "solve" the problem of algorithmic dominance or governance, or being context-aware including being aware of it being too controlling? This is where potentially the pervasive computing community, in a close collaboration with other fields in this truly complex multidisciplinary issue, can contribute a solution to. 


\section{CONCLUSION}

The obvious advantage of the increasing availability of pervasive computing infrastructures is that they will make our lives much easier. However, this evolution also carries on potential risks for individuals and for society as a whole. By blindly accepting the deployment of algorithms that run our society we could become a "dumber" society or even lose control. In this context, "algocracy" may be nontrivial to reconcile with democracy. Dealing with these issues will require deploying a system of societal apparatuses in order to protect the individual citizen against the running code and/or against the potentially malicious usage by individuals of the data that is collected and produced by that code.

In this article, we have explored five avenues. (i) First, there is the obvious attention for data access control. Beside traditional privacy and security concerns, the notion of behavioral privacy will be equally important, as it will be the possibility to understand how the exploitation of our personal data affects algorithms' behavior. (ii) Part of the solution may be to proactively chaperone the ongoing activities of the algocracy with "algorithmic guardians" that can represent and defend us in the algorithmic world. (ii) A less trivial challenge lies in making citizens aware of the code that runs their algocracy and empower them in novel democratic procedures that will be used to manage that code. (iv) We should never give up the possibility for humans to "pull the plug" or to insist on a wisdom of a crowd of (preferably independent) algorithms. (v) Finally, context awareness could be used to "sandbox" the power of certain algorithms in certain contexts and to provide the meta technology to activate and deactivate the sandboxing based on a citizen's expression of discomfort. Finally, we agree with [30] that ethical considerations must be central to new algorithms we will create in the future.

\section{REFERENCES}

[1] F. Zambonelli, "Toward sociotechnical urban superorganisms", Computer, vol. 45, no. 8, pp. 76-78, August 2012.

[2] A. Schmidt, M. Langheinrich, K. Kersting, "Perception beyond the Here and Now", Computer, vol. 44, no. 2, pp. 86-88, Feb. 2011.

[3] S. R. Arnstein, "A ladder of citizen participation", Journal of the American Planning Association, vol. 35, no. 4, pp. 216-224, July 1969.

[4] N. Rodrigues. Algorithmic Governmentality, Smart Cities and Spatial Justice. justice spatiale - spatial justice, Université Paris Ouest Nanterre La Défense, UMR LAVUE 7218, Laboratoire Mosaïques, 2016, Liberty, Equality, IT, 10, http://www.jssj.org/article/gouvernementalite-algorithmique-smartcities-et-justice-spatiale/

[5] Natascha Just, Michael Latzer, "Governance by algorithms: reality construction by algorithmic selection on the Internet”, Media, Culture \& Society, vol 39, Issue 2, pp. 238 - 258, April, 2016

[6] Danilo Doneda, Virgilio A.F. Almeida, "What Is Algorithm Governance?", IEEE Internet Computing, vol. 20 no. 4, p. 60-63, 2016

[7] L. DeNardis, A. M. Hack1, "Internet governance by social media platforms", Telecommunication Policies, vol. 38, no. 9, pp. 761-770, October 2015.

[8] C. Borean, R. Giannantonio, M. Mamei, D. Mana, A. Sassi, F. Zambonelli, "Urban Crowd Steering: An Overview", International Conference on Internet and Distributed Computing Systems, Lecture Notes in Computer Science No. 9258, pp. 143-154, 2015.

[9] A. Maslow, "Higher and Lower Needs", The Journal of Psychology: Interdisciplinary and Applied, Vol. 25, No. 2, 1948.

[10]S. Hajian, F. Bonchi, and C. Castillo. 2016. Algorithmic Bias: From Discrimination Discovery to Fairness-aware Data Mining. In Proceedings of the 22nd ACM SIGKDD International Conference on Knowledge Discovery and Data Mining (KDD '16). ACM, New York, NY, USA, 2125-2126.

[11]S. Wachter-Boettcher. Technically Wrong: Sexist Apps, Biased Algorithms, and Other Threats of Toxic Tech, W.W. Norton \& Company, 2017. 
[12]A. Rouvroy \& B. Stiegler. "The digital regime of truth: from the algorithmic governmentality to a new rule of law", La Deleuziana - On Line Journal of Philosophy, no. 3, pp. 6-29, 2016.

[13]“Geoffrey Hinton, the 'Godfather' of Deep Learning, on AlphaGo.” Accessed July 18, 2016. http://www.macleans.ca/society/science/the-meaning-of-alphago-the-ai-program-that-beat-a-gochamp/.

[14]K. Crawford, "Can an algorithm be agonistic? Ten scenes from life in calculated publics", Science Technology Human Values, p. 162243915589635, Jun. 2015.

[15] "Open Letter on Autonomous Weapons." FLI - Future of Life Institute. Accessed July 18, 2016. http://futureoflife.org/open-letter-autonomous-weapons/.

[16]J.F. Bonnefon, A. Shariff, I. Rahwan, "The social dilemma of autonomous vehicles," Science, vol. 352, no. 6293, pp. 1573-1576, June 2016.

[17]H. Choi, S. Chakraborty, M. Srivastava, "Design and Evaluation of SensorSafe: A Framework for Achieving Behavioral Privacy in Sharing Personal Sensory Information", in Proceedings of TrustCom, Liverpool (UK), June 2012.

[18]P. McDaniel, S. McLaughlin, "Security and Privacy Challenges in Smart Grid", IEEE Security and Privacy, vol. 7, no. 3, pp. 75-77May-June 2009.

[19]J-P. Briot, R.Guerraoui, K-P. Lohr, "Concurrency and distribution in object-oriented programming", ACM Computing Surveys, vol. 30, no. 3, 291-329, September 1998.

[20]T.V. Cutsem, S. Mostinckx, W.D. Meuter, "Linguistic Symbiosis between Event Loop Actors and Threads", Computer Languages, Systems and Structures, vol. 35, no. 1, pp: 80 - 98, 2009.

[21]F.P. Brooks, "No Silver Bullet: Essence and Accidents of Software Engineering", Computer, Vol. 20, No. 4 (April 1987) pp. 10-19.

[22]I. Rahwan. "Society-in-the-loop: programming the algorithmic social contract", Ethics and Information Technology, vol. 2, no. 2, pp. 1572-8439, 2017.

[23]U. Gasser and V. A. F. Almeida, "A Layered Model for AI Governance," IEEE Internet Computing, vol. 21, no. 6, pp. 58-62, November/December 2017.

[24]J.L. Seminara, W.R. Gonzalez, S.O. Parsons, Human factors review of nuclear power plant control room design (Report No. EPRI NP-309). Electric Power Research Institute, Palo Alto, California, 1977.

[25]B. Schilit, N. Adams, R. Want, "Context-aware computing applications", Workshop on Mobile Computing Systems and Applications, 1994, pp. 85-90.

[26]G. D. Abowd, A. K. Dey, P. J. Brown, N. Davies, M. Smith, P. Steggles, "Towards a Better Understanding of Context and Context-Awareness," in Handheld and Ubiquitous Computing, H.-W. Gellersen, Ed. Springer Berlin Heidelberg, 1999, pp. 304-307.

[27]G. Schirner, D. Erdogmus, K. Chowdhury, T. Padir, "The Future of Human-in-the-Loop CyberPhysical Systems," Computer, vol. 46, no. 1, pp. 36-45, Jan. 2013.

[28]S. Krug. Don't make me think: A common sense approach to web usability. Pearson Education India, 2005.

[29]F. Salim and U. Haque, "Urban computing in the wild: A survey on large scale participation and citizen engagement with ubiquitous computing, cyber physical systems, and Internet of Things", International Journal of Human-Computer Studies, vol. 81, pp. 31-48, Elsevier, Sep. 2015.

[30]D. Bianchini and I. Avila, "Smart Cities and Their Smart Decisions: Ethical Considerations", IEEE Technology and Society Magazine, vol. 33, no. 1, pp. 34-40, Spring 2014. 